\title{
Exploring the influence of surface proteins on probiotic activity of Lactobacillus pentosus HC-2 in Litopenaeus vannamei midgut via Label-free quantitative proteomic analysis
}

1 Yang Du ${ }^{\mathrm{a}, \mathrm{b}}$, Han Fang ${ }^{\mathrm{b}, \mathrm{c}}$, Mei Liu ${ }^{\mathrm{a}, \mathrm{b}}$, Keyong Jiang ${ }^{\mathrm{a}, \mathrm{b}}$, Mengqiang Wanga,b, Baojie Wang ${ }^{\mathrm{a}, \mathrm{b} *}$, Lei

2 Wanga,b,

3 a CAS Key Laboratory of Experimental Marine Biology, Institute of Oceanology, Chinese Academy

4 of Sciences, 7 Nanhai Road, Qingdao, 266071, China

$5 \quad{ }^{b}$ Laboratory for Marine Biology and Biotechnology, Qingdao National Laboratory for Marine Science 6 and Technology, Qingdao, 266237, China

7 c University of Chinese Academy of Sciences, 19 Yuquan Road, Beijing, 100049, China

$8{ }^{\mathrm{d}}$ CAS Center for Ocean Mega-Science, Chinese Academy of Sciences, Qingdao 266400, China

$10 *$ *orresponding author: Baojie Wang

11 Email: wangbaojie@qdio.ac.cn.

$12 *$ Co-Corresponding author: Baojie Wang

13 Email: wangbaojie@qdio.ac.cn.

Corresponding/Co-Corresponding authors address: CAS Key Laboratory of Experimental Marine Biology, Institute of Oceanology, Chinese Academy of Sciences, 7 Nanhai Road, Qingdao, 266071, China 


\section{Abstract}

Our previously work showed that Lactobacillus pentosus $\mathrm{HC}-2$ as probiotic could improve the growth performance, immune response, gut bacteria diversity and disease resistance of Litopenaeus vannamei. However, the probiotic mechanism of was not fully characterized. In the present study, histology and proteomic analysis was performed to explore the influence of HC-2 surface protein on its probiotic effects to L. vannamei after feeding the intact surface proteins or the probiotic treated with lithium chloride $(\mathrm{LiCl})$ to remove non-covalently bound surface proteins or no probiotic for four weeks. Histological observation found that feeding with normal HC-2 obviously improved the intestinal histology and enhanced the protective effect against pathogens damages, but fed with LiCl-treated HC2 didn't improve the intestinal environment. A total over 2,764 Peptides and 1,118 uniproteins were identified from L. vannamei midgut, 211 proteins were significant differentially expressed normal HC2 group compared with control, 510 proteins were significant differentially expressed in LiCl-tread HC-2 group compared with control, and 458 proteins were significant differentially expressed in LiCltread HC-2 group compared with normal HC-2 group. GO/KEGG enrichment analysis of the significantly different proteins demonstrated that fed with normal HC-2 mainly induced immune response, metabolic, cell adhesion and cell-cell signaling related proteins up-regulation, which were contributed to bacteria adhesion and colonization in midgut to improve shrimp immune system and growth, but these proteins were suppressed after feeding with deprived surface proteins bacteria. Taken together, these results indicating that the surface proteins were indispensable for HC-2 to execute probiotic effects in midgut of shrimp.

Key words: Litopenaeus vannamei; Lactobacillus pentosus HC-2; Surface proteins; Proteomic analysis. 


\section{Introduction}

Litopenaeus vannamei, is one of the most valuable crustacean aquaculture species worldwide because of its high nutrition value and tolerance to extensive salinity [1]. However, water environment deterioration, frequent disease outbreaks caused by viruses such as WSSV, YHV and IHHNV, or by bacteria like genus Vibrio are more prominent issues as a consequence of the rapidly growing shrimp aquaculture industry $[2,3]$. Thus, there is international concern about dealing with the tough problem by supplying probiotic bacterial cells in food or in the aquatic environment to control the infectious diseases by strengthening the physique of aquatic animals [4, 5]. Among the available probiotics, lactic acid bacteria (LAB) are commonly used and advocated, such as Lactobacillus

pentosus, Lactobacillus helveticus, Lactobacillus delbrueckii, Lactobacillus acidophilus and

Lactobacillusb plantarum have been widely administered for the significant of improving host immune status, strengthening the host digestion, modulating the bacterial community, and antagonizing opportunistic pathogens [6-8].

The mechanisms of probiotic functionality LAB are not completely understood, but it is believed that the maximum probiotic effects can be achieved if the organisms adhere to mucus and/or intestinal epithelial cells [9]. It has recently suggested that surface proteins of lactobacilli bacteria participate in adhesion to epithelial cell lines, gastrointestinal mucins, or extracellular matrix proteins [10-12].

54 Indeed, except mediating binding ability, surface proteins are also involved in maintaining the shape of the bacteria, molecular sieve function, immunomodulation to the host, and providing extracellular enzyme binding sites [13-15]. Due to these proteins bind to the outermost layer of the bacteria with non-covalent bonds, make it possible that using denaturants lithium chloride ( $\mathrm{LiCl}$ ), guanidine hydrochloride $(\mathrm{GuHCl})$, urea or metal chelating agents etc depolymerize them to monomer [16-18]. 
In previous work, we isolated a Lactobacillus pentosus HC-2 strain which has high antimicrobial activity against Vibrio pathogens and adhesive ability to intestinal mucosa, and regulated intestinal flora, and enhanced the growth performance, immune responses, and disease resistance after the $L$. vannamei fed with it [19-21]. The present study aim to further investigate the mediate function of surface proteins of L. pentosus HC-2 in the process of colonization and immune regulation of HC-2 to L. vannamei, label-free proteomic analysis was applied to characterize the proteins expression induced by surface proteins in midgut of shrimp fed with LiCl-treated HC-2.

\section{Materials and methods}

\section{Bacterial growth and surface proteins shaving}

Lactobacillus pentosus HC-2 (GenBank Accession No. KU995298) was previously isolated from the intestinal tract of fish (Acanthogobius hasta) by our laboratory [21], which was saved in - 80 ${ }^{\circ} \mathrm{C}$ in de Man, Rogosa, and Sharpe (MRS) broth containing 20\% (v/v) glycerol. After the recovery, the bacteria were cultured unstirred in MRS medium at $37^{\circ} \mathrm{C}$ under anaerobic conditions.

Cell surface proteins shaving performed as previously [22]. Briefly, 500-mL culture of bacteria on the transition between late exponential and stationary phase (OD600 $\approx 1.7)$ were harvested centrifugation $\left(3,000 \times \mathrm{g}, 10 \mathrm{~min}, 4^{\circ} \mathrm{C}\right)$. Then, the cell pellets were washed three times with 1 M phosphate-buffered saline (PBS) containing 25\% sucrose. After centrifugation, the bacteria cells were incubating the cells in $25 \mathrm{ml}$ of $5 \mathrm{M} \mathrm{LiCl}$ to stripe the surface associated proteins. After treatments, cells were collected and washed three times with autoclave sterilized seawater.

\section{Feeding trials}

The experimental diets were prepared as previously that bacteria were resuspended in sterilized 
seawater and sprayed on basal commercial feed (containing crude protein $42 \%$, crude fat $7 \%$, ash $15 \%$, and water $11 \%)$ at $5 \times 10^{8}$ colony-forming units (CFU) g/feed [22]. A total of 600 shrimps $(3.5 \pm 0.06$

g) were grown in twelve aquaria $(60 \mathrm{~L})$, each containing 50 shrimp. The experiments were designed as follows: C group, shrimp fed a basal commercial diet alone as the control; R group, shrimp fed a basal commercial diet + normal HC-2; L group, shrimp fed a basal commercial diet + LiCl treated HC2. Three replicates were set in each feeding group. Keeping the fresh seawater (salinity, 30\%o) at $30 \pm$ $2{ }^{\circ} \mathrm{C}$ with continuous aeration and a $50 \%$ water change every day. Animals were fed three times per day, and the daily feeding rate was $10 \%$ of the body weight.

\section{Challenge test}

After the feeding experiment, 25 shrimp were random selected from each aquarium and transferred to a tank with $30 \mathrm{~L}$ of seawater for challenge test. The live Vibrio parahaemolyticus E1 ATCC 17802 Strains was used for challenge, which was cultured aerobically in 2216E broth (Qingdao Hope BiolTechnology Co., Ltd) at $28^{\circ} \mathrm{C}$ for $18 \mathrm{~h}$. Preliminary experiment showed the appropriate bacteria dose was $10^{7} \mathrm{CFU} / \mathrm{mL}$. During the challenge experiment, the shrimp were fed with basical diet.

\section{Histology of the Midgut}

The histology determine was carried out as described in Sha et al. (2016) [20]. Five shrimps were freely selected from each treatment group upon termination of the feeding and challenge experiments and sampled the midguts to dissect and fix (60\% absolute ethanol, 30\% trichloromethane, $10 \%$ acetic acid) for $19 \mathrm{~h}$. Following, the fixed tissues were dehydrated in ascending concentrations of alcohol $(70,80,95$, and $100 \%)$, cleared in toluene, embedded in paraffin, and sectioned at $10 \mu \mathrm{m}$ with a rotary microtome. The sectioned tissues were stained with hematoxylin and eosin, and images were obtained 
with a light microscope.

\section{Protein extraction and separation by $1 D$ gel}

Upon termination of the feeding experiment, the midguts of twenty shrimps from each treatment group were dissected and the intestinal contents was removed by flushing with sterile pre-cooled PBS. Total intestine proteins were extracted as the method described by Sengupta et al. (2011) [23] with some modifications. Pooled samples $(1 \mathrm{~g})$ were thoroughly grind into fine powder in liquid nitrogen with mortar and pestle and dissolved in $5 \mathrm{~mL}$ extraction buffer $(0.5 \mathrm{M}$ Tris- $\mathrm{HCl}(\mathrm{pH} 7.5), 0.7 \mathrm{M}$ sucrose, of Tris-phenol and shaking for $30 \mathrm{~min}$, the upper phenolic phase was collected by centrifuging ( 8000 protein precipitation, four volumes of $0.1 \mathrm{M}$ ammonium acetate in methanol were added and kept overnight at $-20^{\circ} \mathrm{C}$. Protein pellet was collected after centrifugation at $8000 \times \mathrm{g}$ for $10 \mathrm{~min}$ at $4{ }^{\circ} \mathrm{C}$, and washed thrice with ice-cold acetone at $4^{\circ} \mathrm{C}$. The pellet was dried in vacuum for $2 \mathrm{~h}$ and then solubilized in $100 \mu \mathrm{L}$ rehydration solution $(8 \mathrm{M}(\mathrm{w} / \mathrm{v})$ urea, $0.1 \mathrm{M}(\mathrm{w} / \mathrm{v})$ Tris, $10 \mathrm{mM}$ DTT). The concentration of protein was determined using the Bradford method [24]. Finally, proteins were loaded on 10\% SDS-

PAGE and separated at $120 \mathrm{~V}$ for $2 \mathrm{~h}$, and visualized using colloidal Coomassie Blue after electrophoresis.

\section{Trypsin in-gel digestion}

Protein gels were washed thrice with $50 \%$ acetonitrile $(\mathrm{ACN}) / 50 \% \mathrm{NH}_{4} \mathrm{HCO}_{3}(100 \mathrm{mM})$ for 10 
acetonitrile (ACN). Then, alkylation was performed with $55 \mathrm{mM}$ iodoacetamide (Sigma-Aldrich)/50 mM NH4HCO3 (pH 8.0) for $30 \mathrm{~min}$ in the dark. The gel bands were alternately washed twice with 10 mM NH4HCO3 and 100\% ACN, respectively. The gels were dried by Speed-Vac and digested with trypsin $(0.01 \mu \mathrm{g} / \mu \mathrm{l})$ (Promega, Madison, WI) in $10 \mathrm{mM} \mathrm{NH4HCO3}$ at $37^{\circ} \mathrm{C}$ overnight. Digestion was stopped by adding $60 \% \mathrm{ACN} / 5 \%$ formic acid (FA) solution.

\section{Nanoflow liquid chromatography-tandem mass spectrometry}

Prior to analyse the tryptic digest extracts using Thermo Scientic EASY-nLC 1000 System (Nano HPLC), the crude polypeptides were firstly desalted with a ChromXP Trap column (Nano LC TRAP Column, $3 \mu \mathrm{m} \mathrm{C}_{18}-\mathrm{CL}, 120 \mathrm{~A}, 350 \mu \mathrm{m} \times 0.5 \mathrm{~mm}$, Foster City, CA, USA), and then eluted onto a second analytical column of Nano $\mathrm{LC} \mathrm{C}_{18}$ reversed-phase column $\left(3 \mathrm{C}_{18}-\mathrm{CL}, 75 \mu \mathrm{m} \times 15 \mathrm{~cm}\right.$, Foster City, CA, USA) under a linear gradient formed by mobile phases A (5\% ACN and 0.1\% FA) and B (95\% CAN and $0.1 \% \mathrm{FA}$ ) at a flow rate of $300 \mathrm{~nL} / \mathrm{min}$ for $120 \mathrm{~min}$. Triple TOF $5600 \mathrm{MS}$ (Foster City, CA, USA) was performed to automatically switch the TOF-MS and production acquisition in data-dependent mode by Analyst (R) Software (TF1.6).

\section{Protein identification}

Three biological replicates were performed for the control, R and L group. The LC-MS/MS raw data were processed using the MaxQuant (version 1.5.2.8) for peptide/protein identification and quantification. MS/MS spectra was searched by the Andromeda search engine using a database consisting 28,384 sequences of the shrimp transcriptome, downloaded from NCBI. Search parameters were as follows: monoisotopic mass values; enzyme was trypsin; static Modification with $\mathrm{C}$ carboxyamidomethylation (57.021 Da); dynamic Modification was M Oxidation (15.995Da); 
143 Precursor ion mass tolerance $\pm 15 \mathrm{ppm}$; Fragment ion mass tolerance with $\pm 20 \mathrm{mmu}$; allowance of 144 two missed cleavage site; false discovery rate (FDR) set as 0.01 . Peptides identification with $95 \%$ 145 confidence are considered "significant sequences". For protein quantification, a minimum of 146 two ratio counts was set to compare and normalize protein intensities across runs [25]. The absolute 147 abundance of different proteins were then calculated using the intensity-based absolute quantification 148 (iBAQ) algorithm, and iBAQ data were used for the t-test [26].

\section{Bioinformatics analysis} three ontologies analysis, namely molecular functions (MF), cellular components (CC), and biological process (BP) [27]. Subcellular localisation for each protein was predicted according to GO annotation by Uniprot software (http://www.uniprot.org/). GO items without corresponding annotation were first deleted from the protein table, and then the IDs of listed proteins were plotted at the BP, CC, and MF levels. In addition, differentially expressed proteins (fold changes $>1.5, p<0.05$ ) were mapped to the GO database, and the number of proteins at each GO term was computed. The results from label159 free proteomics were used as the target list. The background list was generated by downloading the 160 GO database.

\section{Quantitative real-time PCR}

RNA was prepared from midguts of ten L. vannamei that had been used for Real-time PCR 
GA, USA) and reverse-transcripted into first-strand cDNA using the RevertAid First Strand cDNA Synthesis Kit (Thermo Fisher Scientific) according to the manufacturer's instructions. Seven proteins including hemocyanin (Hem), C1q-binding protein (Clq), calreticulin (Cal), pyruvate kinase 2 (Pyr), integrin (Int), proliferating cell nuclear antigen (Pro), hemocyte transglutaminase (Htr) were selected to determine their mRNA levels, all of which were annotated to $L$. vannamei and were important proteins functioning in immune response, metoblic, adhesion and cell-cell signaling. The primer sequences were designed by Primer 5 (S1 Table). The reactions were carried out using Bio-Rad IQTM5 real-time PCR with a total volume of $20 \mathrm{~m} \mathrm{~L}(2 *$ SYBR Green Mix (Vazyme Biotech): $10 \mathrm{~m} \mathrm{~L}$, Primer: $1.0 \mathrm{~m} \mathrm{~L}$, cDNA template: $5.0 \mathrm{~m} \mathrm{~L}$, and PCR grade water: $4.0 \mathrm{~m} \mathrm{~L}$ ). The qRT-PCR procedure was as follows: initial denaturation at $95{ }^{\circ} \mathrm{C}$ for $2 \mathrm{~min}$; 40 cycles of amplification $\left(95^{\circ} \mathrm{C}\right.$ for $15 \mathrm{~s}, 60{ }^{\circ} \mathrm{C}$ for $20 \mathrm{~s}$, and $72{ }^{\circ} \mathrm{C}$ for $\left.20 \mathrm{~s}\right)$. The cycle threshold $(\mathrm{Ct})$ was measured, and the relative gene expression was calculated using the $2^{-\Delta \Delta \mathrm{Ct}}$ method. $\beta$-actin gene was used as endogenous control. Three biological replicates and three technical replicates were done for all PCR experiments, and significance was determined at the $\mathrm{P}<0.05$.

\section{Results and discussion}

\section{Histology of the Midgut}

To investigate the effects of dietary Licl-treated probiotics on the midgut of the shrimp, a histological study was performed at the end of the feeding experiment and challenge assay as shown in Fig 1. Compared with the control group, the mucosae of $\mathrm{R}$ group improved more dense and more bloom (Fig 1B), but the mucosae of L group shrimps displayed to thin and loose (Fig 1C). After the shrimps were challenged by the $V$. parahaemolyticus $\mathrm{E} 1$, the mucosae shed and piled in the intestinal lumen, and the lamina propria exposed and appeared loose in the control and L groups (Figs 1D and 
1F), especially, some individuals showed some reduced folding of the digestive epithelium in the crease. However, the shrimps fed with normal HC-2 appeared to no signs of necrotic enterocytes or cell damage (Fig 1E).

\section{Label-free proteomic analysis of intestine proteins of $L$. vannamei}

In total, 2,810 proteins were detected. The differential protein expression among the three groups as shown in S2 Appendix, and proteins with fold change $\geq \pm 1.5$ and $\mathrm{P}<0.05$ were considered to be significantly differentially abundant. Pairwise comparison of intestinal protein with different levels among R/control, L/control, and L/R are illustrated in Fig 2, identified 210, 510 and 458 differentially abundant proteins, respectively. The numbers of upregulated proteins were 110, 134, and 85, respectively, whereas 101,376 , and 373 proteins, respectively, were down-regulated. The relationships among the experimental groups was performed by a PCoA analysis in proteins expression patter form.

Samples of C, R and L group were clustered independently (Fig 3). To comprehensively analyze the impact of HC-2 and LiCl-treated HC-2 had on protein expression changes, the differentially abundant proteins were subjected to cluster analysis under different experimental conditions (Fig 4). Heat map showed that samples of C, R and L group were clustered respectively. The results of the heat map and PCoA were consistent to some extent, indicating that the proteins expression in $\mathrm{C}, \mathrm{R}$ and $\mathrm{L}$ groups differed and that the proteins expression in the midgut was influenced by the addition of HC-2 and LiCl-treated HC-2.

\section{GO analysis of DEPs in L. vannamei midguts}

Based on the gene ontology (GO) analysis in level 2 of biological process, cellular components and molecular functions associated with the significantly differentially abundant intestinal proteins (q- 
value $<0.05$, and $\log 2 \mid$ fold change $\mid>1.5$ ) (Fig 5). Among the 210 differentially abundant proteins in the $\mathrm{R} /$ control comparison, 93 proteins played a role in 23 different biological processes, 139 proteins were related to cellular component and 46 proteins had distinct molecular functions. Compared with the control group, 510 differentially abundant proteins in the L group comprised 189 proteins that participated in 25 biological processes, 100 proteins had specific molecular functions, and 318 proteins were related to cellular components. Biological process analysis indicated that the transport, signal transduction, reproduction, immune system process, protein transport, transmembrane transport, embryo development, cell cycle, cell death, carbohydrate metabolic process, vesicle-mediated transport, growth, protein targeting, cell-cell signaling and cell adhesion processes involved the majority of proteins in $\mathrm{R} /$ control or L/control comparison. Cellular component analysis of R/control and $\mathrm{L} /$ control comparison revealed that the main differentially abundant proteins belonged to cytoplasm, membrane, nucleus, plasma membrane, mitochondrion, cytoskeleton, extracellular region and endoplasmic reticulum. Molecular function analysis revealed that most differentially abundant proteins were related to metal Ion binding and transmembrane transporter activity in both the $\mathrm{R} /$ control and L/control comparisons. Comparing $\mathrm{L}$ to $\mathrm{R}, 177$ proteins played roles in the biological processes of transport, reproduction, signal transduction, vesicle-mediated transport, cell cycle, protein transport, immune system process, embryo development, growth, cell death, carbohydrate metabolic process, cell motility, cell-cell signaling, cell division, developmental maturation, protein targeting, membrane organization, cell adhesion; the cellular component of 290 differentially abundant proteins were cytoplasm, membrane, nucleus, mitochondrion, cytoskeleton, plasma membrane, extracellular region, 
molecular functions categories were related to metal ion binding, transmembrane transporter activity and signal transducer activity.

\section{KEGG pathway analysis of the DEPs in L. vannamei midguts}

KEGG pathway analysis was performed to determine the biological pathways that involved the

differentially abundant proteins (q-value $<0.05$, and $\log 2 \mid$ fold change $\mid>1.5$ ) induced by HC-2 and

LiCl-treated HC-2 treatments fed in the diet (Fig 6). The DEGs between R group and the control group mainly enriched in mTOR signaling pathway, ECM-receptor interaction, RNA degradation, Apoptosis,

control group mainly enriched in Protein processing in endoplasmic reticulum, RNA transport,

Tyrosine metabolism, Arginine and proline metabolism, Lysosome, mRNA surveillance pathway,

Cysteine and methionine metabolism and Glutathione metabolism. The DEGs between R and L group mainly enriched in Protein processing in endoplasmic reticulum, Endocytosis, Ribosome, Lysosome,

Glycolysis/Gluconeogenesis, Cysteine and methionine metabolism and Tyrosine metabolism.

Proteins potentially involved in shrimp immune response, metabolic, cell-adhesion and cell-

The GO enrichment analysis of biological process of the significantly different proteins involved

244 in shrimp immune system process, cell-cell signaling process, cell adhesion and carbohydrate metabolic process among the three groups as shown in $\mathrm{S} 3 \mathrm{Table} 1$. In the $\mathrm{R} /$ control group, 10 proteins involved in immune system process, 7 proteins involved in cell-cell signaling process, 5 proteins involved in cell adhesion and 4 proteins involved in carbohydrate metabolic process were significantly increased, and 3, 5 proteins involved in immune and carbohydrate metabolic process were significantly 
decreased. Among them, tyrosine-tRNA ligase, C1q-binding protein, tyrosine-protein phosphatase

69D-like and Neutral alpha-glucosidase AB were most up-regulated in the four process that the

expression level reached 10.28, 5.66, 2.88 and 6.77 fold, respectively. However, in the L/control group,

there didn't induce more proteins up-regulation, and 22, 12, 6 and 18 proteins were participated in

immune, cell-cell signaling, cell adhesion and carbohydrate metabolic process respectively were

significantly down-regulated.

Based on the KEGG enrichment analysis, several of the proteins that were differentially expressed

in shrimp fed with probiotic are involved in immune system process (mTOR signaling pathway,

257 Apoptosis, Phagosome, Oxidative phosphorylation, MAPK signaling pathway, Lysosome, Protein 258 processing in endoplasmic reticulum), Metabolism process (Arginine and proline metabolism, 259 Tyrosine metabolism, Glutathione metabolism, Glycerolipid metabolism/Histidine metabolism, 260 Cysteine and methionine metabolism, Fatty acid metabolism, Carbon metabolism), cell adhesion 261 process (Focal adhesion, Tight junction, ECM-receptor interaction), and Cell signaling process 262 (Calcium signaling pathway, Oxytocin signaling pathway, FoxO signaling pathway and Wnt signaling pathway) (S3 Table 2).

\section{Analysis of selected proteins affected by HC-2 and LiCl-treated HC-2 treatments}

To validate the label-free based proteomic results, quantitative real-time PCR was used to analyze

the transcripts of proteins found to be differentially abundant after HC-2 and LiCl-treated HC-2 treatments (Fig 7). The qPCR results showed that three proteins (Int, Pro and Htr) expressed higher than determined in $\mathrm{R}$ group proteome, the other proteins were consistent with the proteomics data, 


\section{Discussion}

Gastro-intestinal tract, the most important digestive and absorption organ in shrimps, where residing a large number of microorganisms with complex structures. These organisms depend on and restrict each other with hosts, and forming a unique intestinal micro-ecosystem in the long process of evolution [28]. Recent years, it is widely recognized that supplement with probiotics in aquaculture may stabilize the indigenous microflora, and normalize the host-microbe interaction, which is contribute to reduce the incidence of diseases [29]. Our previous work demonstrated that L. pentosus HC-2 has ideal probiotic effect to L. vannamei, but the probiotic action of surface components of HC-2 to shrimp is not clear. This work, to investigate the impact of surface proteins on probiotic effect of HC-2 to L. vannamei, proteomic analyses were conducted to using a label-free based LC-MS/MS approach to obtain protein data from three biological replicates.

Several studies demonstrated that dietary probiotic supplementation could improve the growth performance which was deemed to be attributed to intestinal physiology changes and gut epithelium morphology changes [30,31], such as an improved intestinal microvillus structure and a greater absorptive surface area $[32,33]$. In the present study, the changes in the intestinal microvilli and the folding of the digestive epithelium varied between dietary groups, and obvious improvement in intestinal histology was observed after shrimp fed with the normal probiotic HC-2, and the intestinal tissue was not damaged after the shrimp were challenged by $V$. parahaemolyticus E1. These results are similar to the findings of Merrifield et al. (2010) [33], who found that Pediococcus acidilactici fed fish had significantly longer microvilli than other groups of fish, but are contrary to the findings of Sha et al. (2016) [20], who reported that dietary HC-2 didn't improve the intestinal morphology of L. vannamei. These differently phenomena may be attributable to the bacteria concentration used in 
the dietary is too low $\left(10^{7} \mathrm{CFU} / \mathrm{g}\right)$ than in this work $\left(5 \times 10^{8} \mathrm{CFU} / \mathrm{g}\right)$, which hinder the $\mathrm{HC}-2$ to be the dominant microflora in the shrimp intestines to improve the intestinal morphology. However, no signs improvement in intestinal histology post the shrimp fed with LiCl-treated HC-2, instead, even to be more badly compared with the control shrimp that the mucosae showed to thin and loose after the shrimp challenged by pathogens. This results indicated that the surface proteins play important roles in probiotic function of HC-2 to improve the gut physiology and morphology.

With the intensive development of aquaculture and the frequent outbreaks of disease, varied probiotics have been developed to meet the demand of pollution-free immune enhancer. In shrimp farming, many authors have studied the influence of probiotic on the immune response. For example, Wang et al. (2010) [34] indicated that fed with Lactobacillus enhanced shrimp growth performance, increased digestive enzyme activities, and promoted non-specific immunity. Zheng et al. (2017) [35] also revealed that the administration of Lactobacillus pentosus AS13 effectively improved the shrimp growth performance, feed utilization, digestive enzymes and disease resistance. In present work, the proteomic analysis showed that fed with the normal HC-2 induced the proteins involved in immune system process (mTOR signaling pathway, Apoptosis, Phagosome, Oxidative phosphorylation, MAPK signaling pathway, Lysosome, Protein processing in endoplasmic reticulum) up-regulation, but many immune-related proteins were down-regulation in LiCl-treated HC-2 group shrimp midgut, which suggesting surface proteins play vital roles in mediation HC-2 enhance the shrimp intestinal immune response.

Several available genomic information descripted the metabolic activities of lactobacilli, which indicated that surface proteins are importance of carbohydrate metabolism in the host [36]. It has been reported that Lactobacillus paracasei or Lactobacillus rhamnosus probiotics supplementation 
of HBF mice exerted microbiome modification and resulted in altered hepatic lipid metabolism coupled with lowered plasma lipoprotein levels and apparent stimulated glycolysis, and also affected diverse range of metabolism pathways including amino-acid metabolism, methylamines and SCFAs [37]. In the present study, we found some proteins involved in carbohydrate metabolic process were significantly up-regulation after shrimp fed with normal HC-2, but proteins participated in other metabolic pathway including Arginine and proline, Tyrosine, Glutathione, Glycerolipid/Histidine, Cysteine and methionine, Fatty acid metabolism expressed insignificance. While, feeding with LiCltreated HC-2 led to the overall downregulation of these metabolism related proteins, which indicated that surface proteins are importance in HC-2 regulation and maintenance of the shrimp intestinal metabolic.

Adhesion is the interaction of the bacteria surface structure (adhesin) attached with the surface receptors on the epithelial cells of the host, is the first step of bacterial colonization, and is the key for bacteria to grow, reproduce and functional exercise. Recent studies have indicated that the attachment of bacteria including the hydrophobicity and self-agglutination of the bacterial surface, lipoteichoicacid (LTA), exopolysaccharides (EPS) and related cell surface proteins to mucosal surfaces is the initial event in intestinal adhesion and colonization $[38,16]$. Meanwhile, there are many surface proteins that mediate adhesion in lactobacillus have been reported, such as CmbA/Lar_0958, EF-Tu, GAPDH, GroEL, Lam29, MapA, MBF, Msa , Mub (Mub family), Pili, 32-Mmubp, FbpA and GroEL, etc [39-41]. Probiotics adhere to host intestinal mucus, intestinal epithelial cells, extracellular stroma by means of its surface proteins, and/or other bacteria lipodesmoic acid to effectively prevent pathogenic infection [42-44]. In present study, many related cell adhesion proteins were displayed significantly up-regulation in shrimp midgut after feeding with the normal HC-2, but the LiCl-treated 
HC-2 induced many cell adhesion proteins significantly down-regulation. Besides the adhesion ability, the surface proteins were studied have important functions in cell-cell signaling process, and interaction with the host immune system or environment [45]. In this study, we found that the proteins involved in cell-cell signaling pathway were up-regulated in shrimp midgut after fed with the normal HC-2, but the fed with surface proteins shaving bacteria induced these proteins decreased. These results indicated that surface proteins play crucial role in adhesion and colonization of HC-2 in the shrimp midgut, and were contributed to activation of a series of molecular signals communication with the surface cell of host.

In conclusion, fed with normal HC-2 obviously improved the intestinal histology and enhanced the protective effect against pathogens damages, but fed with LiCl-treated HC-2 didn't improve the intestinal structure. GO and KEGG enrichment analysis of significantly proteins in R/control and

$\mathrm{L} /$ control indicated that most proteins were involved in immune system process, metabolic process, adhesion process, and cell-cell signaling process. However, these proteins were significantly upregulation in shrimp midgut after feeding the normal HC-2, and were significantly down-regulation in shrimp fed with LiCl-treated HC-2. The results in present work indicated that surface proteins play an important roles in mediation of HC-2 to improve intestinal histology, immune response, metabolic, adhesion and signaling communication in midgut of shrimp, which might provide a base data to understand the probiotic mechanism excised by HC-2.

\section{Supporting information}

355 S1 Appendix. Primers. (PDF)

S2 Appendix. Different expression proteins (Excel) 


\section{Acknowledgments}

We thank Dr Wenbin Zhan (Laboratory of Pathology and Immunology of Aquatic Animals,

Ocean University of China), for providing $V$. parahaemolyticus E1, and we are grateful to all the

laboratory members for their technical advice and helpful suggestions.

\section{References}

[1] Ji P-F, Yao C-L, Wang Z-Y. Two types of calmodulin play different roles in Pacific white shrimp (Litopenaeus vannamei) defenses against Vibrio parahaemolyticus and WSSV infection. Fish Shellfish Immunol. Elsevier; 2011; 31: 260-268.

[2] Lightner D V. Virus diseases of farmed shrimp in the Western Hemisphere (the Americas): a review. J Invertebr Pathol. Elsevier; 2011; 106: 110-130.

[3] Li F, Xiang J. Signaling pathways regulating innate immune responses in shrimp. Fish Shellfish Immunol. Elsevier; 2013; 34: 973-980.

[4] Verschuere L, Rombaut G, Sorgeloos P, Verstraete W. Probiotic bacteria as biological control agents in aquaculture. Microbiol Mol Biol Rev. Am Soc Microbiol; 2000; 64: 655-671.

[5] Dawood MAO, Koshio S, Abdel-Daim MM, Van Doan H. Probiotic application for sustainable aquaculture. Rev Aquac. Wiley Online Library; 2018;

[6] Yousefian M, Amiri MS. A review of the use of prebiotic in aquaculture for fish and shrimp. African J Biotechnol. Academic Journals (Kenya); 2009; 8.

[7] Ige BA. Probiotics use in intensive fish farming. African J Microbiol Res. Academic Journals; 2013; 7: 2701-2711. 
[8] Maeda M, Shibata A, Biswas G, Korenaga H, Kono T, Itami T, et al. Isolation of lactic acid bacteria from kuruma shrimp (Marsupenaeus japonicus) intestine and assessment of immunomodulatory role of a selected strain as probiotic. Mar Biotechnol. Springer; 2014; 16: 181-192.

[9] Kesarcodi-Watson A, Kaspar H, Lategan MJ, Gibson L. Probiotics in aquaculture: the need, principles and mechanisms of action and screening processes. Aquaculture. Elsevier; 2008; 274: $1-14$.

[10] Heinemann C, van Hylckama Vlieg JET, Janssen DB, Busscher HJ, van der Mei HC, Reid G. Purification and characterization of a surface-binding protein from Lactobacillus fermentum RC14 that inhibits adhesion of Enterococcus faecalis 1131. FEMS Microbiol Lett. Blackwell Publishing Ltd Oxford, UK; 2000; 190: 177-180.

[11] Rojas M, Ascencio F, Conway PL. Purification and characterization of a surface protein from Lactobacillus fermentum 104R that binds to porcine small intestinal mucus and gastric mucin. Appl Environ Microbiol. Am Soc Microbiol; 2002; 68: 2330-2336.

[12] Roos S, Jonsson H. A high-molecular-mass cell-surface protein from Lactobacillus reuteri 1063 adheres to mucus components. Microbiology. Microbiology Society; 2002; 148: 433-442.

[13] Jakava-Viljanen M, Palva A. Isolation of surface (S) layer protein carrying Lactobacillus species from porcine intestine and faeces and characterization of their adhesion properties to different host tissues. Vet Microbiol. Elsevier; 2007; 124: 264-273.

[14] Chen X, Xu J, Shuai J, Chen J, Zhang Z, Fang W. The S-layer proteins of Lactobacillus crispatus strain ZJ001 is responsible for competitive exclusion against Escherichia coli O157: H7 and Salmonella typhimurium. Int J Food Microbiol. Elsevier; 2007; 115: 307-312. 
[15] Konstantinov SR, Smidt H, De Vos WM, Bruijns SCM, Singh SK, Valence F, et al. S layer protein A of Lactobacillus acidophilus NCFM regulates immature dendritic cell and T cell functions. Proc Natl Acad Sci. National Acad Sciences; 2008; pnas-0810305105.

[16] Kos B, Šušković J, Vuković S, Šimpraga M, Frece J, Matošić S. Adhesion and aggregation ability of probiotic strain Lactobacillus acidophilus M92. J Appl Microbiol. Wiley Online Library; 2003; 94: 981-987.

[17] der Mei HC, de Belt-Gritter B, Pouwels PH, Martinez B, Busscher HJ. Cell surface hydrophobicity is conveyed by S-layer proteins-a study in recombinant lactobacilli. Colloids Surfaces B Biointerfaces. Elsevier; 2003; 28: 127-134.

[18] Smit E, Oling F, Demel R, Martinez B, Pouwels PH. The S-layer protein of Lactobacillus acidophilus ATCC 4356: identification and characterisation of domains responsible for Sprotein assembly and cell wall binding. J Mol Biol. Elsevier; 2001; 305: 245-257.

[19] Sha Y, Wang B, Liu M, Jiang K, Wang L. Interaction between Lactobacillus pentosus HC-2 and Vibrio parahaemolyticus E1 in Litopenaeus vannamei in vivo and in vitro. Aquaculture. Elsevier; 2016; 465: 117-123.

[20] Sha Y, Liu M, Wang B, Jiang K, Qi C, Wang L. Bacterial population in intestines of litopenaeus vannamei fed different probiotics or probiotic supernatant. J Microbiol Biotechnol. 2016; 26: $1736-1745$.

[21] Sha Y, Wang L, Liu M, Jiang K, Xin F, Wang B. Effects of lactic acid bacteria and the corresponding supernatant on the survival, growth performance, immune response and disease resistance of Litopenaeus vannamei. Aquaculture. Elsevier; 2016; 452: 28-36. 
[22] Du Y, Zhou S, Liu M, Wang B, Jiang K, Fang H, et al. Understanding the roles of surface proteins in regulation of Lactobacillus pentosus $\mathrm{HC}-2$ to immune response and bacterial diversity in midgut of Litopenaeus vannamei. Fish Shellfish Immunol. Elsevier; 2019; 86: 1194-1206.

[23] Sengupta D, Kannan M, Reddy AR. A root proteomics-based insight reveals dynamic regulation of root proteins under progressive drought stress and recovery in Vigna radiata (L.) Wilczek. Planta. Springer; 2011; 233: 1111-1127.

[24] Bradford MM. A rapid and sensitive method for the quantitation of microgram quantities of protein utilizing the principle of protein-dye binding. Anal Biochem. Elsevier; 1976; 72: 248254.

[25] Cox J, Michalski A, Mann M. Software lock mass by two-dimensional minimization of peptide mass errors. J Am Soc Mass Spectrom. Springer; 2011; 22: 1373-1380.

[26] Luber CA, Cox J, Lauterbach H, Fancke B, Selbach M, Tschopp J, et al. Quantitative proteomics reveals subset-specific viral recognition in dendritic cells. Immunity. Elsevier; 2010; 32: 279289.

[27] Consortium GO. The Gene Ontology (GO) database and informatics resource. Nucleic Acids Res. Oxford University Press; 2004; 32: D258--D261.

[28] Cardona E, Gueguen Y, Magré K, Lorgeoux B, Piquemal D, Pierrat F, et al. Bacterial community characterization of water and intestine of the shrimp Litopenaeus stylirostris in a biofloc system. BMC Microbiol. BioMed Central; 2016;16: 157.

[29] Lara-Flores M. The use of probiotic in aquaculture: an overview. Int Res J Microbiol. 2011;2: 471-478. 
441

[30] Daniels CL, Merrifield DL, Boothroyd DP, Davies SJ, Factor JR, Arnold KE. Effect of dietary Bacillus spp. and mannan oligosaccharides (MOS) on European lobster (Homarus gammarus L.) larvae growth performance, gut morphology and gut microbiota. Aquaculture. Elsevier; 2010; 304: 49-57.

[31] Li P, Burr GS, Gatlin III DM, Hume ME, Patnaik S, Castille FL, et al. Dietary supplementation of short-chain fructooligosaccharides influences gastrointestinal microbiota composition and immunity characteristics of Pacific white shrimp, Litopenaeus vannamei, cultured in a recirculating system. J Nutr. Oxford University Press; 2007; 137: 2763-2768.

[32] de Rodrigáñez MA, Diaz-Rosales P, Chabrillón M, Smidt H, Arijo S, León-Rubio JM, et al. Effect of dietary administration of probiotics on growth and intestine functionality of juvenile Senegalese sole (Solea senegalensis, Kaup 1858). Aquac Nutr. Wiley Online Library; 2009;15: $177-185$.

[33] Merrifield DL, Harper GM, Dimitroglou A, Ringø E, Davies SJ. Possible influence of probiotic adhesion to intestinal mucosa on the activity and morphology of rainbow trout (Oncorhynchus mykiss) enterocytes. Aquac Res. Wiley Online Library; 2010; 41: 1268-1272.

[34] Wang G, Huang Y, Zhou Y, Dong S, Huang W, Yan Q, et al. Effects of Lactobacillus on growth performance, digestive enzyme activities and non-specific immunity of Litopenaeus vannamei. Chinese J Anim Nutr. Chinese Association of Animal Science and Veterinary Medicine; 2010; 22: $228-234$.

[35] Zheng CN, Wang W. Effects of Lactobacillus pentosus on the growth performance, digestive enzyme and disease resistance of white shrimp, Litopenaeus vannamei (Boone, 1931). Aquac Res. Wiley Online Library; 2017; 48: 2767-2777. 
[36] Lebeer S, Vanderleyden J, De Keersmaecker SCJ. Genes and molecules of lactobacilli supporting probiotic action. Microbiol Mol Biol Rev. Am Soc Microbiol; 2008; 72: 728-764.

[37] Martin F-PJ, Wang Y, Sprenger N, Yap IKS, Lundstedt T, Lek P, et al. Probiotic modulation of symbiotic gut microbial-host metabolic interactions in a humanized microbiome mouse model. Mol Syst Biol. EMBO Press; 2008; 4: 157.

[38] Granato D, Perotti F, Masserey I, Rouvet M, Golliard M, Servin A, et al. Cell surface-associated lipoteichoic acid acts as an adhesion factor for attachment of Lactobacillus johnsoniiLal to human enterocyte-like Caco-2 cells. Appl Environ Microbiol. Am Soc Microbiol; 1999; 65: 1071-1077.

[39] Nishiyama K, Sugiyama M, Mukai T. Adhesion properties of lactic acid bacteria on intestinal mucin. Microorganisms. Multidisciplinary Digital Publishing Institute; 2016; 4: 34.

[40] Buck BL, Altermann E, Svingerud T, Klaenhammer TR. Functional analysis of putative adhesion factors in Lactobacillus acidophilus NCFM. Appl Environ Microbiol. Am Soc Microbiol; 2005; 71: 8344-8351.

[41] Bergonzelli GE, Granato D, Pridmore RD, Marvin-Guy LF, Donnicola D, Corthésy-Theulaz IE. GroEL of Lactobacillus johnsonii La1 (NCC 533) is cell surface associated: potential role in interactions with the host and the gastric pathogen Helicobacter pylori. Infect Immun. Am Soc Microbiol; 2006; 74: 425-434.

[42] Hynönen U, Westerlund-Wikström B, Palva A, Korhonen TK. Identification by flagellum display of an epithelial cell-and fibronectin-binding function in the SlpA surface protein of Lactobacillus brevis. J Bacteriol. Am Soc Microbiol; 2002; 184: 3360-3367. 
484 [43] Johnson-Henry KC, Hagen KE, Gordonpour M, Tompkins TA, Sherman PM. Surface-layer protein extracts from Lactobacillus helveticus inhibit enterohaemorrhagic Escherichia coli O157: H7 adhesion to epithelial cells. Cell Microbiol. Wiley Online Library; 2007; 9: 356-367.

487 [44] Antikainen J, Anton L, Sillanpää J, Korhonen TK. Domains in the S-layer protein CbsA of Lactobacillus crispatus involved in adherence to collagens, laminin and lipoteichoic acids and in self-assembly. Mol Microbiol. Wiley Online Library; 2002; 46: 381-394.

490 [45] Kaper JB, Sperandio V. Bacterial cell-to-cell signaling in the gastrointestinal tract. Infect Immun. Am Soc Microbiol; 2005; 73: 3197-3209. 


\section{Figure caption.}

493 Fig 1. Histology with hematoxylin and eosin staining of the shrimp midguts after feeding with different

494 diets for 4 weeks. Images A, B, C, D, E and F are arbitrarily chosen examples of the histology observed 495 in three groups. A: Gut histology of shrimps were fed a basic diet; B: Gut histology of shrimps were 496 fed a basic diet supplied with normal L. pentosus HC-2; C: Gut histology of shrimps were fed a basic 497 diet supplied with LiCl-treated L. pentosus HC-2; D, E and F were showed the gut histology of shrimps 498 in A, B and C respectively which were challenged by Vibrio parahaemolyticus E1. LP: lamina propria, 499 M: mucosae, MV: microvilli, SCE: surface cell epithelium. Bar: $100 \mu \mathrm{m}$.

Fig 2. Volcano plot of changes in the levels of identified intestine proteins of shrimp analyzed using label-free quantitative proteomics after feeding with different diets. Note: $\mathrm{C}$, shrimps were fed with basal diet; R, shrimps were fed with basal diet supplemented with normal L. pentosus HC-2; L, shrimps were fed with basal diet supplemented with LiCl-treated L. pentosus HC-2 (L).

Fig 3. Principal coordinates analysis scores based on the Unifrac distance. PC1: the first principle component; PC2: the second principle component. Shrimps were fed a basal diet (C) or a basal diet supplemented with L. pentosus HC-2 (R), LiCl-treated L. pentosus HC-2 (L).

Fig 4. Heat map of the proteins expression diversity among the three groups ( 9 samples). Shrimps were fed a basal diet $(\mathrm{C}[\mathrm{C} 1, \mathrm{C} 2, \mathrm{C} 3])$ or a basal diet supplemented with normal L. pentosus $\mathrm{HC}-2$ (R 
510 Fig 5. Functional categorization based on gene ontology (GO) in biological process, cellular 511 components and molecular funcitons level analysis of significantly differentially abundant intestineal 512 proteins. Shrimps were fed a basal diet (C) or a basal diet supplemented with L. pentosus HC-2 (R), 513 LiCl-treated L. pentosus HC-2 (L).

514 Fig 6. Distribution of differentially abundant proteins in shrimp midgut in KEGG pathways (Top 20).

515 Note: Shrimps were fed a basal diet (C) or a basal diet supplemented with L. pentosus HC-2 (R), LiCl516 treated L. pentosus HC-2 (L).

517 Fig 7. Validation analysis of label-free proteomics using quantitative real-time PCR to determine the 518 selected proteins expression in midgut of $L$. vannamei. Note: $\mathrm{R}$, shrimps were fed with basal diet 519 supplemented with normal L. pentosus HC-2; L, shrimps were fed with basal diet supplemented with 520 LiCl-treated L. pentosus HC-2 (L). Fold changes of proteins expression represent experimental group compared with control group. 


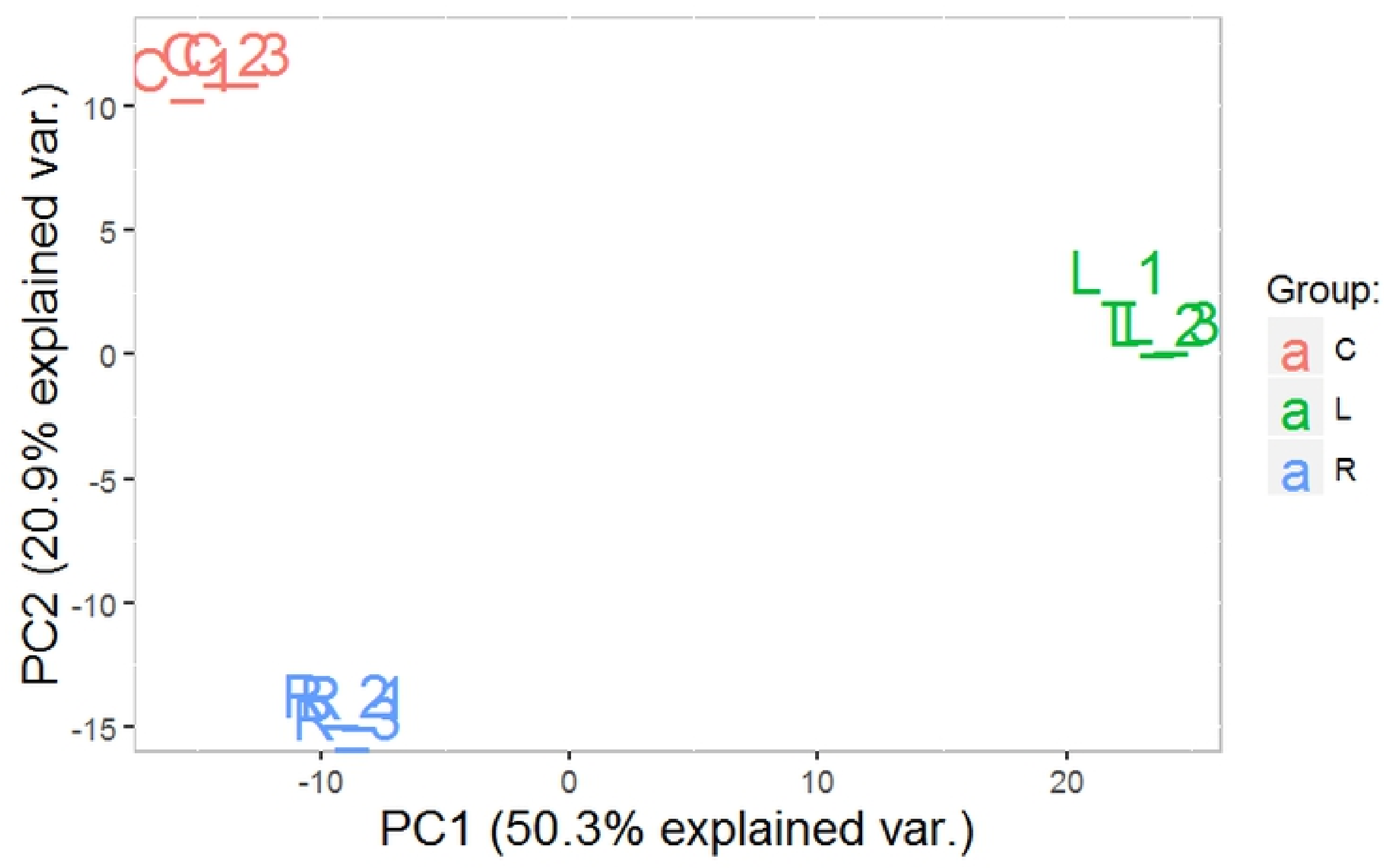

Figure 


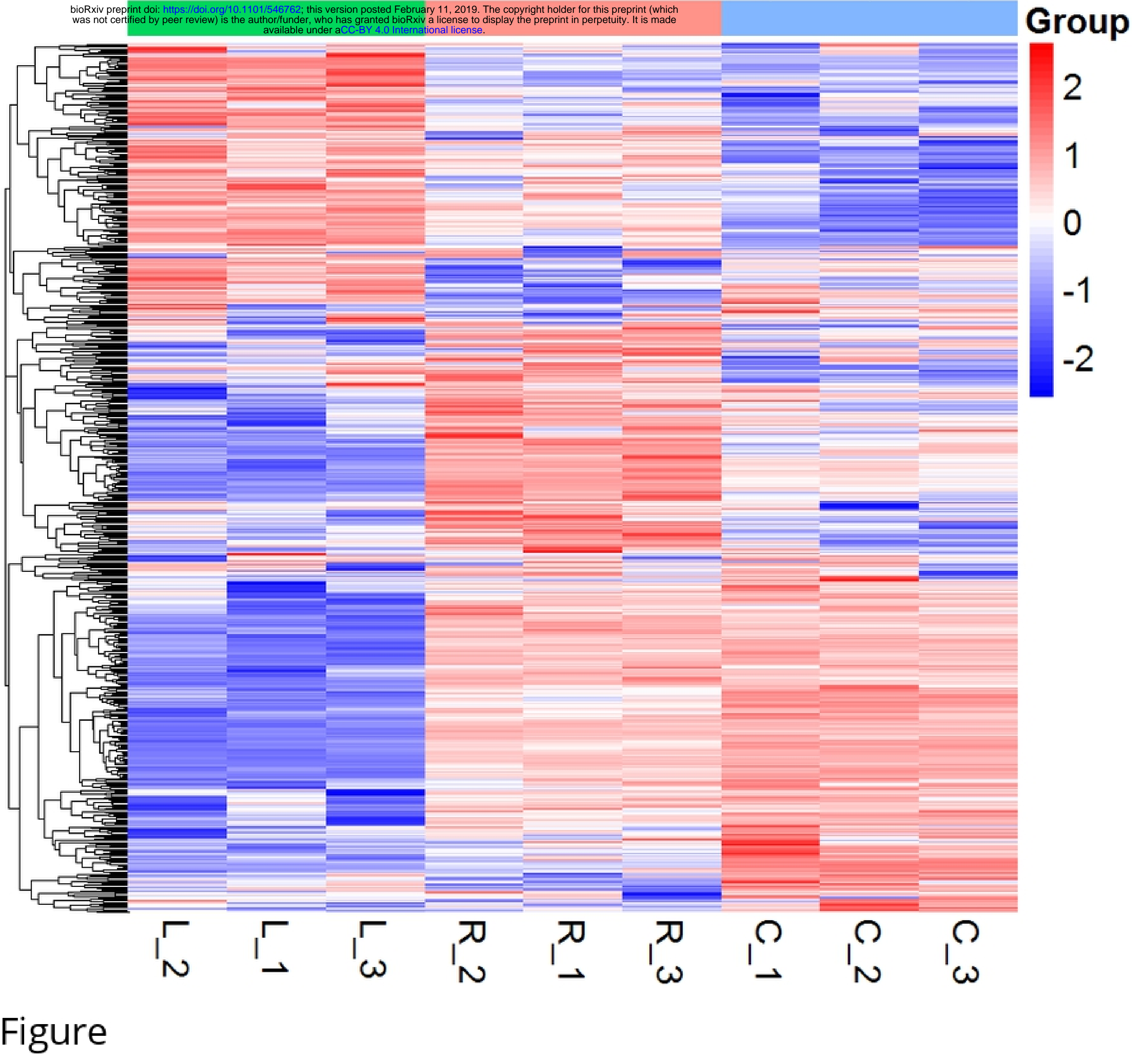




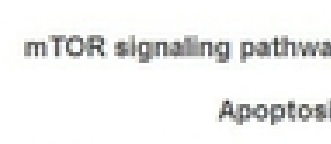

ECM-receptor interaction -

RNA degradation

Phagosome

Butanoate metabolism

Synthesis and degradation of ketone bodies.

Other types of O-glycan biosynthesis

Protein export

Inositol phosphate metabolism

Fructose and mannose metabolism

FoxO signaling pathway

Alanine, aspartate and glutamate metabolism
Retinol metabolism

Alanine, aspartate and glutamate metabolism
Retinol metabolism -

Pentose and glucuronate interconversions

One carbon pool by folate

Aminoacyl-tRNA blosynthesis

Oxidative phosphorylation

Porphyrin and chlorophyll metabolism

$$
0.2
$$

0.4

\section{Ratio}

(P2) L-vs-C KEGG pathway enrichment

bioRxiv preprint doi: https://doi.org/10.1101/546762; this version pos bioRxiv preprint doi: https://doi.org/10.1101/546762; this version posted February 11,2019 . The copyright holder for this preprint (which
was not certified by peer review) is therauthorffunder, who has granted bioRxiv a license to display the preprint in perpetuity. It is made

Arginine and proline metabolism

Tyrosine metabolism

mRNA surveillance pathway

Ascorbate and aldarate metabolism

Glutathione metabolism

Sulfur metabolism

Metabolism of xenobioties by eytochrome P450

Histidine metabolism

Glyeosaminoglyean degradation

Glycerolipid metabolism

Cysteine and methionine metabolism

Lysosome

AGE-RAGE signaling pathway in diabetic complications

Pentose and glucuronate interconversions

RNA transport

Protein processing in endoplasmic reticulum

Selenocompound metabolism

Wnt signaling pathway

0.7
Gene number

- 1

?

3

5

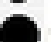

$\log 10$ (Pvalue)

1.6

of

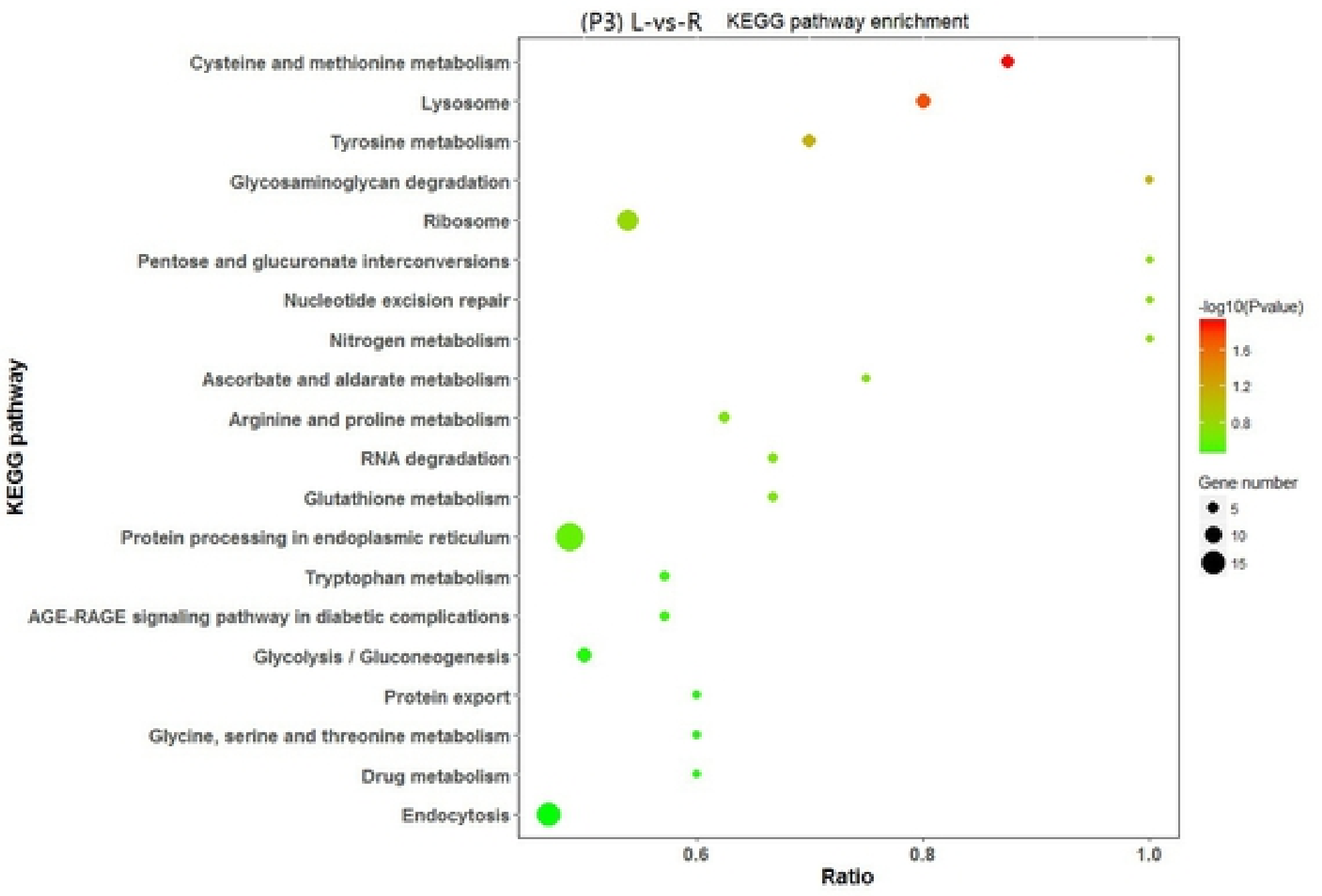

Figure 

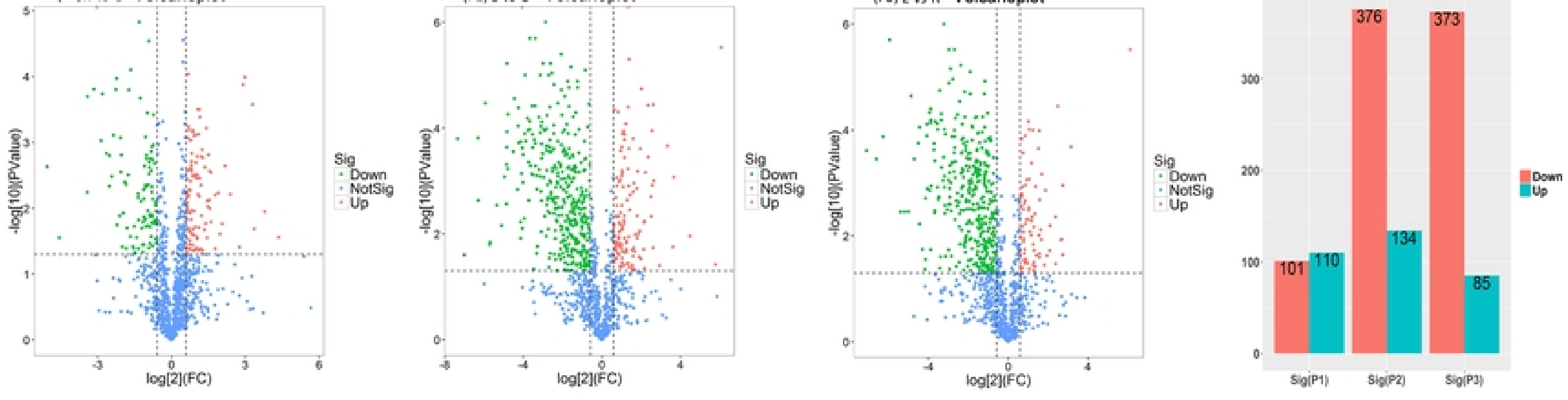

Figure 
(P1) GO enrichment analysis R-vs-C

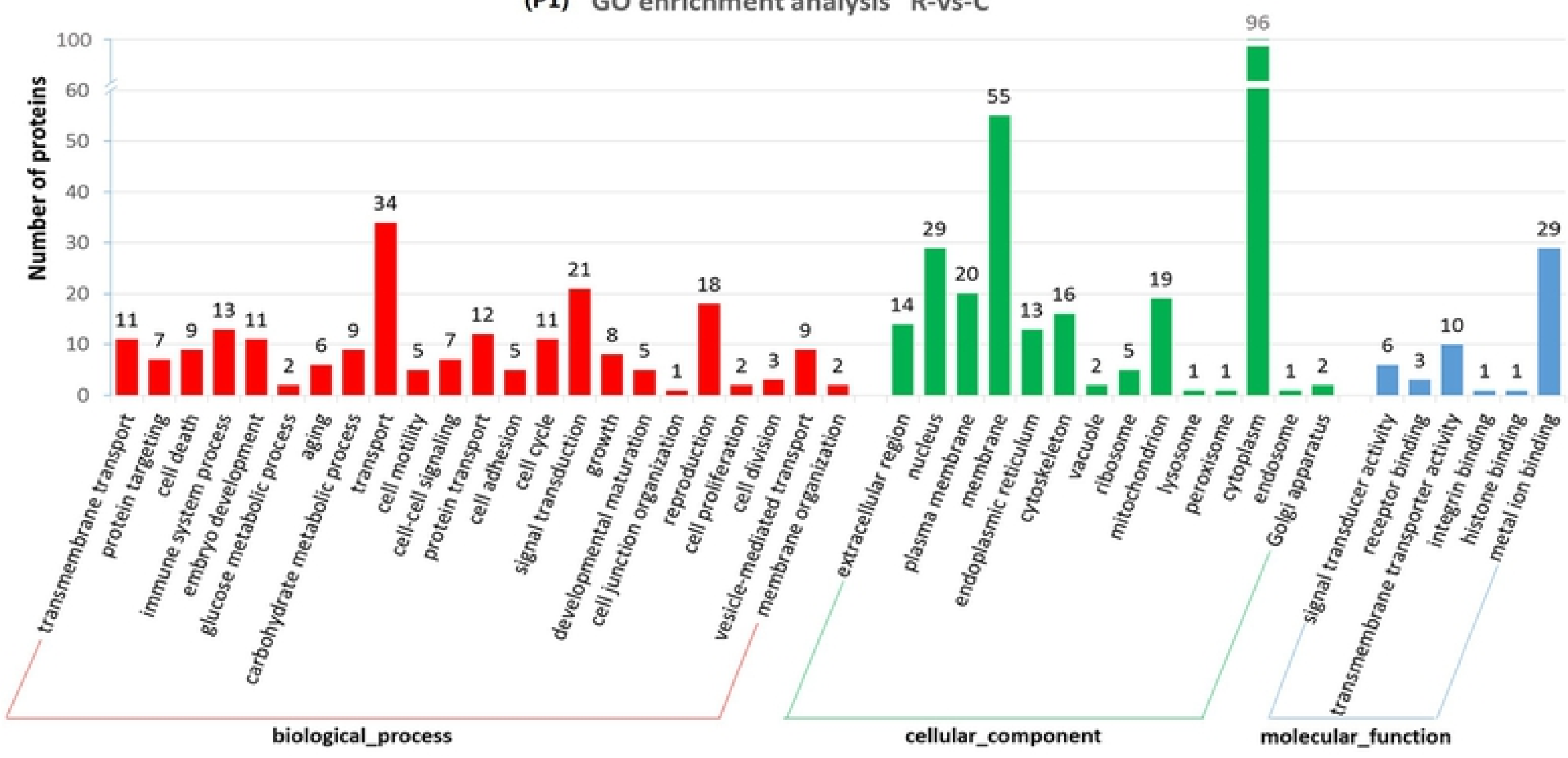

(P2) GO enrichment analysis L-vs-C

bioRxiv preprint doi: https://doi.org/10.1101/546762; this version posted February 11,2019 . The copyright holder for this preprint (which
was not certified by peer review) is the author/funder, who has granted bioRxiv a license to display the preprint in perpetuity. It is made
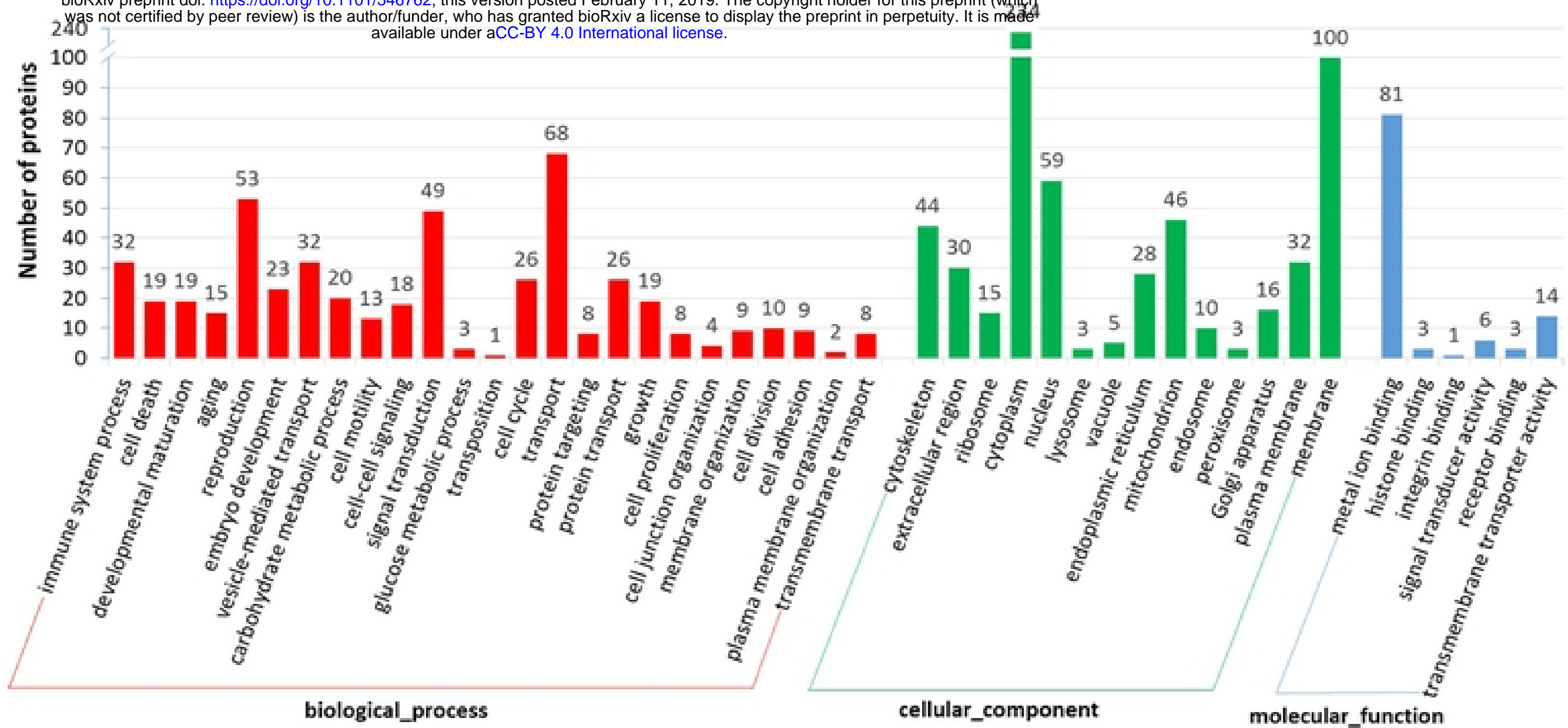

(P3) GO enrichment analysis L-vs-R
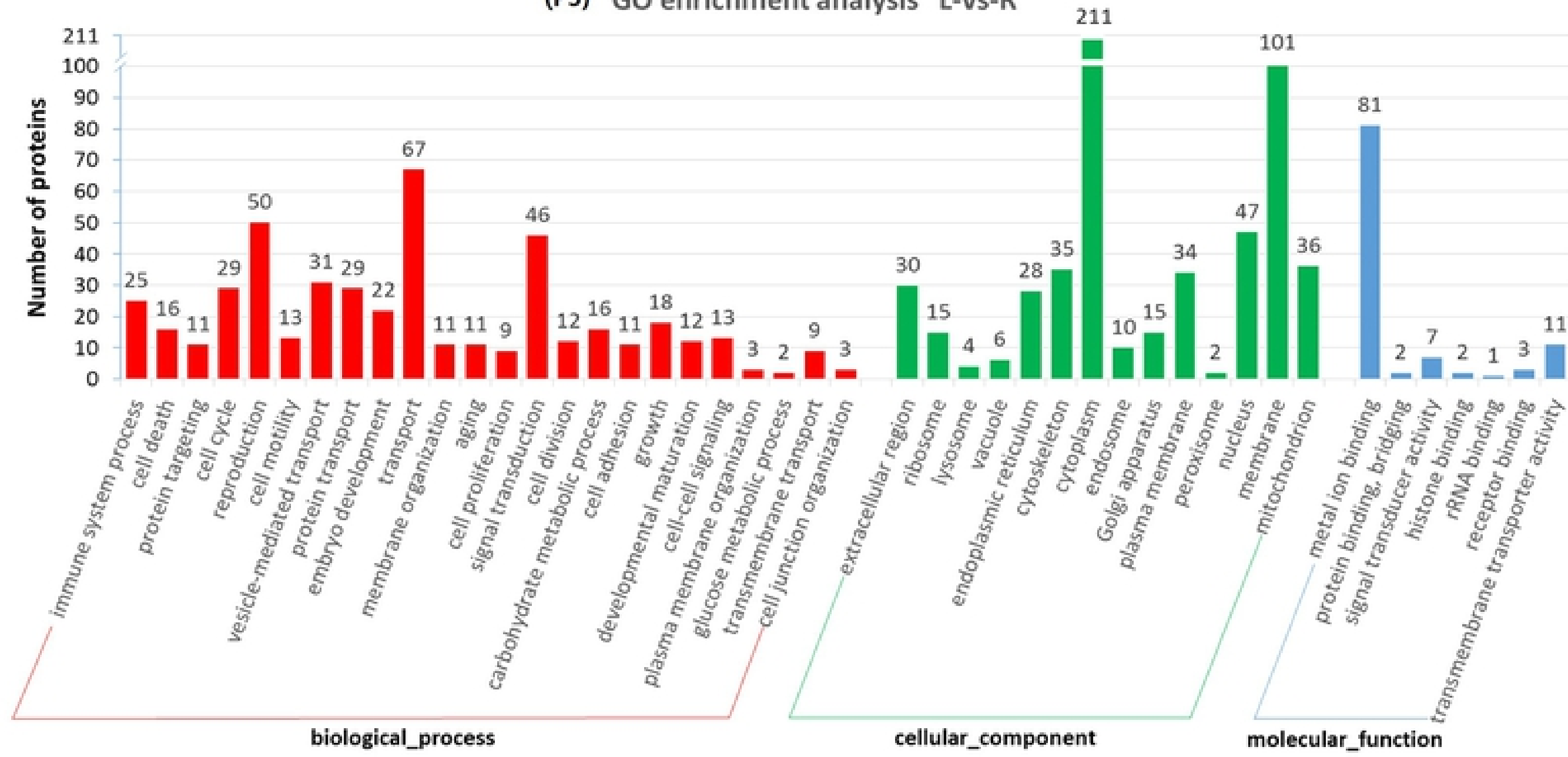

\section{Figure}

